\title{
Topochemical photopolymerization of diolefin crystals
}

\author{
Masaki Hasegawa \\ Department of Synthetic Chemistry, Faculty of Engineering, \\ The University of Tokyo, Hongo, Bunkyo-ku, Tokyo 113, Japan
}

\begin{abstract}
Topochemical [2+2] photocyclo-dimerization and -polymerization of olefinic derivative crystals are reviewed from the points of view of synthetic and structural chemistries. In 1964 Schmidt and co-workers established the topochemical concept concerning to the photoreactive olefinic crystals: the topochemical reaction tends to occur with a minimum of atomic and molecular motion. The first example of topochemical polymerization (four-center type photopolymerization) was found in 1967, where 2,5-distyrylpyrazine crystal affords highly crystalline linear polymer, repeating intermolecular [2+2] photocyclo-dimerization of two olefin bonds in a molecule with retention of crystal symmetry. Almost all of the reaction behaviors and configurations of the product are interpreted in terms of topochemical principle (reactive double bonds are arranged in parallel with a distance within $4.2 \AA$ ) while several exceptional examples have been recently reported.
\end{abstract}

\begin{abstract}
A great variety of reactivities and photoproducts has been revealed in the four-center type photopolymerization of unsymmetric diolefin crystals, depending on a slight modification of chemical structure or crystallization process of the monomer. A single type of dimer is formed exclusively and accumulated at the intermediate stage, and only the reactive species having even-numbered degree of polymerization participates in chain growing process. Optically active polymers and polymers with different substituents in alternative order can be prepared from the unsymmetric diolefin crystals.
\end{abstract}

\section{INTRODUCTION}

A great number of crystalline state organic photoreactions have been reported so far in which several examples date a hundred years back. Among these reactions [2+2] photocyclodimerization of olefinic derivative crystals is the most commonly encountered reaction. For example, the photodimerization of cinnamic acid crystals was reported by Lieberman in 1889 (ref. 1), and in 1943 , formation of $\alpha$-truxillic and $\beta$-truxinic acids from two types of cinnamic acid crystals was interpreted as a crystal lattice controlled reaction by Bernstein and Quimby (ref. 2). In 1964, Schmidt and coworkers correlated the crystal structures of cinnamic acid and several other enone derivatives with the steric configurations of their photoproducts (ref. 3 ). The result established the topochemical concept in which the reaction tends to occur with a minimum of atomic and molecular motion. More concretely, the crystal structures of cinnamic derivatives were divided into three types: the $\alpha$-type crystal in which the double bonds of neighboring molecules are arranged in parallel and make contact at a distance of 3.6-4.1 $\AA$ across a center of symmetry; the $\beta$-type, characterized by a lattice having one axial length of 3.9-4.1 $\AA$ between translationally related molecules; and the $\gamma$-type, in which no double bonds of neighboring molecules are within $4.7 \AA$.

\section{$\underline{\alpha-t y p e ~ c r y s t a l}$}
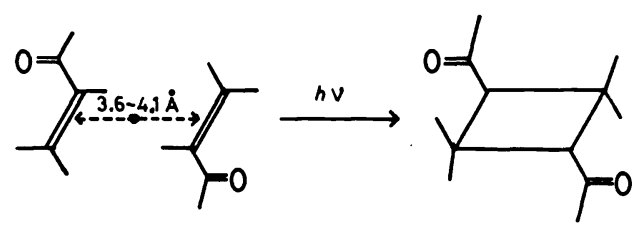

B-type crystal

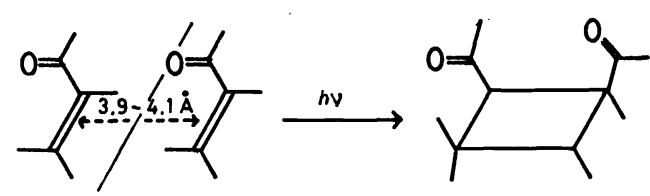


On photoirradiation of cinnamic derivatives, for example, an $\alpha$-type crystal gives a centrosymmetric dimer related to $\alpha$-truxillic derivative (I-dimer)*, $\beta$-type crystal gives a dimer of mirror symmetry related to $\beta$-truxinic derivative (m-dimer), and a $\gamma$-type crystal is photostable.

Topochemical polymerization was first discovered in the reaction of 2,5-distyrylpyrazine (DSP) crystal in 1967 (ref. 4). On photoirradiation, extremely highly crystalline poly-DSP is formed, step by step, by repeating [2+2] photocyodimerization between intermolecular double bonds in DSP crystal.

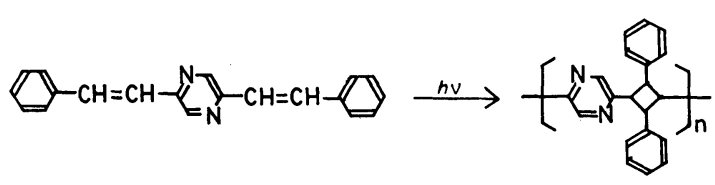

DSP

Poly-DSP

The crystallographic studies revealed that the polymerization of DSP crystal followed the topochemical rule enunciated by Schmidt: intermolecular reactive double bonds in DSP crystal, are arranged in parallel at a distance of 3.939 $\AA$ across a center of symmetry ( $\alpha$-type crystal). Furthermore, it was demonstrated that the unit cell of DSP crystal was strictly duplicated to that of poly-DSP crystal with retention of crystal symmetry during the reaction (ref. 5). The topochemical photopolymerization of DSP was the first example of an organic reaction in which crystal-to-crystal transformation was visualized; it was named four-center type photopolymerization.

TABLE 1. Crystal data of DSP $(\alpha \text { and } \gamma)^{*} 1$ and poly-DSP

\begin{tabular}{|c|c|c|c|c|c|c|c|}
\hline & $\begin{array}{l}\text { Space } \\
\text { group }\end{array}$ & $a, \stackrel{\circ}{\AA}$ & $\mathrm{b}, \stackrel{\circ}{\mathrm{A}}$ & c, $\stackrel{\circ}{\AA}$ & $\mathrm{z}$ & Dx & $\begin{array}{c}\mathrm{C}-\mathrm{C} \\
\text { distance, }\end{array}$ \\
\hline $\begin{array}{l}\operatorname{DSP}(\alpha) \\
\operatorname{DSP}(\gamma)\end{array}$ & $\begin{array}{l}\mathrm{Pbca} \\
\mathrm{P} 21 / \mathrm{a}\end{array}$ & $\begin{array}{l}20.638 \\
13.833\end{array}$ & $\begin{array}{c}9.599 \\
18.615 \\
(92.63) \star 2\end{array}$ & $\begin{array}{l}7.655 \\
5.823\end{array}$ & $\begin{array}{l}4 \\
4\end{array}$ & $\begin{array}{l}1.244 \\
1.261\end{array}$ & $\begin{array}{l}3.939 \\
4.187 \\
4.369\end{array}$ \\
\hline poly-DSP & $\mathrm{Pbca}^{\star} 3$ & 18.36 & 10.88 & 7.52 & 4 & 1.257 & --- \\
\hline
\end{tabular}

* 1 Photoreactive $\alpha$-type crystal of DSP is formed readily by recrystallization from benzene solution, while a considerable portion of photostable crystal ( $\gamma$-type) is produced when the crystal was formed by sublimation (ref. 6).

$* 2$ B $\left({ }^{\circ}\right)$.

* 3 One paper reported erroneously that the group of poly-DSP is $\mathrm{P} 2{ }_{1} \mathrm{ca}$ (ref. 7). We reinvestigated the crystal structure and reconfirmed the retention of space group (Pbca) during the reaction (ref. 8 ).

\section{FUNDAMENTALS OF FOUR-CENTER TYPE PHOTOPOLYMERIZATION}

Further synthetic study of four-center type photopolymerization established the empirical rule that the crystalline state [2+2] photodimerization of

Diolefinic monomers

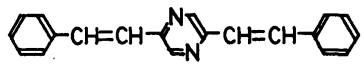

DSP series

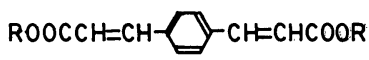

p-PDA series

$\mathrm{ROOC}(\mathrm{CN})=\mathrm{CH}-\mathrm{CH}=\mathrm{C}(\mathrm{ON}) \mathrm{COOR}$

p-CPA series
[2+2] Photodimerizations ( Model reactions)

$$
\text { stilbazol }
$$
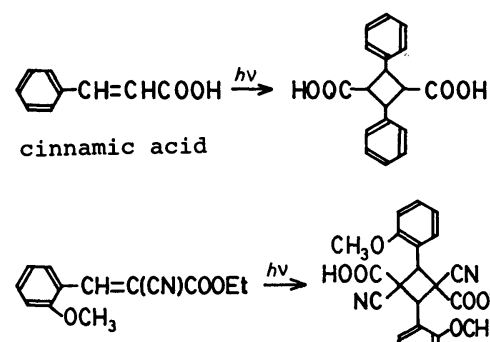
$\alpha$-cyanocinnamic derivative

* I-dimer structure is tentatively assigned to the product by spectroscopic analysis, although the dimer has not yet been isolated. 
olefins can be widely extended to the crystalline state photopolymerization of diolefins with a rigid linear structure having the conjuated double bonds separated by the 1,4-position of an aromatic ring, such as stilbazole to DSP series, cinnamic acid to the p-PDA series (dialkyl 1,4-phenylenediacrylates), and $\alpha$-cyanocinnamic acid derivative to the $p-C P A$ series (dialkyl $\alpha, \alpha^{\prime}-$ dicyano-1,4-phenylenediacrylates) (ref. 9).

Since then, according to this empirical rule, a great number of photoreactive diolefinic crystals have been found. For example the photoreactive unsymmetric diolefins which consist of two different photodimerizable olefinic units in a molecule, were prepared based on this rule and converted to the chiral oligomeric photoproducts (ref. 10). As was expected from the empirical rule, a commonly appearing intermolecular affinity in photoreactive olefins during the crystallization was explained by the observed unusually close contact between aromatic ring and electron-rich moiety in these crystals, such as between phenylene ring and carbonyl oxygens in diethyl 1,4 -phenylendiacrylate crystal (ref. 11).

All the photoreactive diolefin crystals, which have been reported to give the high polymers in high yields, are related to the center of symmetry (centrosymmetric $\alpha$-type crystal). Thus these diolefin crystals afford a polymer having the recurring cyclobutane structure with a 1,3 -trans configuration and 1,4-arylene units alternatively in the chain (ref. 12), (Fig. 1).

The reaction readily affords a highly crystalline linear polymer quantitatively as long as the polymerization temperature is sufficiently low to maintain the rigidity of reacting crystal lattice. Therefore, at the same conversion, a higher reduced viscosity is generally observed for the product polymerized at the lower temperature.

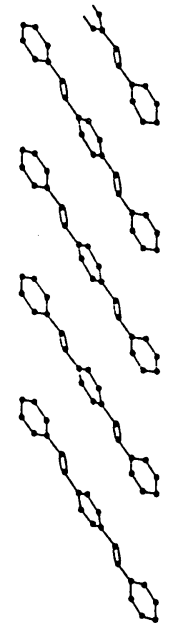

(a)

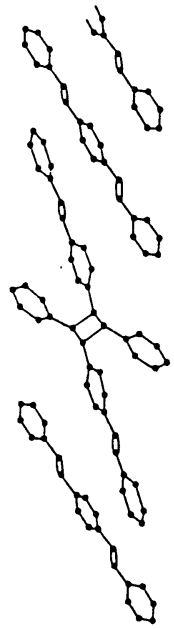

(b)

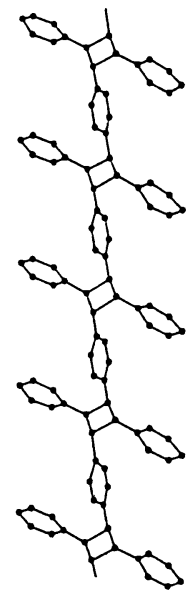

(c)

Fig. 1. Schematic illustration of the conversion of (a) monomer DSP into (b) dimer and (c) polymer.

For the last five years, anomalous topochemical behaviors which deviate from the accepted topochemical rule have been reported on several olefin crystals (ref. 13). Recently in author's laboratory various types of photoreactive unsymmetric diolefin crystals were prepared and investigated on their photochemical behavior. As a result, several outstanding variations of topochemical behavior caused by a slight modification of chemical structures were observed, and characteristic features of the photochemical behavior of unsymmetric diolefin crystals were visualized (ref. 14).

In the present article topochemical [2+2] photocycloaddition polymerizations are reviewed, focusing on recent research results, from the points of view of synthetic and structural chemistries.

\section{DEVIATIONS FROM TOPOCHEMICAL PRINCIPLE}

Recent studies of the [2+2] photocyclodimerization of olefinic crystals have shown several examples which deviate from the accepted rule of the topochemical reactions enunciated by schmidt.

In 1982 Ramamurthy et al. reported that the reactive double bonds of 7methoxycoumarin were rotated by $65^{\circ}$ with respect to each other, with centerto-center distance between the double bonds being $3.83 \AA$. The reaction was the first clear-cut example of topochemical reaction between non-parallel double bonds (ref. 15). Similar results were reported with respect to the 2,5-dibenzylidenecyclopentanone crystal (ref. 16). These results have been interpreted in terms of the distance between the molecular planes of reactive double bonds as well as the double bond axis. 
Another type of anomalous topochemical photodimerizations has been reported of 7-chlorocoumarin (C-C distance $4.454 \AA$ ) (ref. 17), and of the vitamine $A_{1}$ (intermolecular C-C distance of double bonds in side chain $4.459 \AA$ ) (ref. 18). Although these distances are beyond the accepted limit of $3.5-4.2 \AA$, these double bonds undergo topochemical photocyclodimerization. Contrary to these results, the mixed crystal of $1: 1$ donor-acceptor type cinnamic acids is reported to be photostable, in which double bonds of adjacent donor and acceptor components in the stack are in parallel and within a photoreactive distance of each other (3.80 ) (ref. 19).

We have also observed exceptional photocyclodimerizations of several olefin crystals, such as 4-formyl cinnamic acid (1, distance of double bonds 4.825 \&) (ref. 20), methyl 4-(3-oxo-3-phenyl-1-propenyl) benzoate (C-C distance $4.407 \AA)$ (ref. 21 ), and 1,4-dicinnamoyl benzene (2) (ref. 22 ).

The reactive $\mathrm{C}-\mathrm{C}$ distance of double bonds in 1 crystal represents the known maximum record to date. Photocyclodimerization behavior of 1 , 3,4-dichloro, and several other cinnamic acid derivatives is greatly influenced by other molecules out side of the crystal (ref. 23, 24). For example, B-type crystal of 1 , on photoirradiation at room temperature in the presence of even a trace of moisture, dimerizes to crystalline m-dimer containing one molecule of water. The continuous change of the $\mathrm{x}$-ray diffraction pattern during the photodimerization indicates a typical crystal-to-crystal transformation process. On the other hand, the same crystal photodimerizes into amorphous m-dimer in the absence of water. In both reactions, the final yield is nearly quantitative.

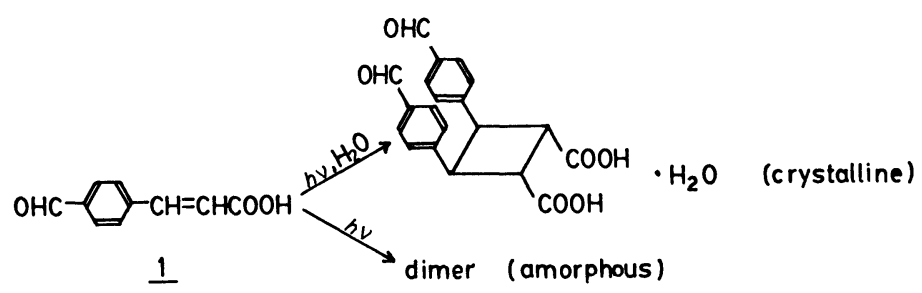

In the crystal of 1,4-dicinnamoylbenzene ( 2 ), the distances between the intermolecular photoadductive carbons are 3.973 and $4.086 \AA$ for one cyclobutane ring, and 3.903 and $3.955 \AA$ for the other. The two topochemical pathways may occur competitively in a single crystal of 2 at the initial stage of reaction. Then, both intramolecular photocyclodimerization and intermolecular photopolymerization of diolefinic mono-cyclobutane

intermediate (3) occurs competitively to give tricyclic dimer $21,22,23,24-$ tetraphenyl-1, $4,11,14$-tetraoxo-2(13),12(13)-diethano [ 4.4] paracyclophene (4) or oligomers. The formation of a $\delta$-type cyclobutane ring, which was confirmed by NMR analysis and by crystal structure of 2 , is extremely rare in the crystaliine state reaction; only one example has been previously reported on 1,1'-(tri-methylene) bisthymine (ref. 25). However, the photodimerization of $\underline{2}$ is the first report in which $\delta$-type cyclobutane deriverative is produced
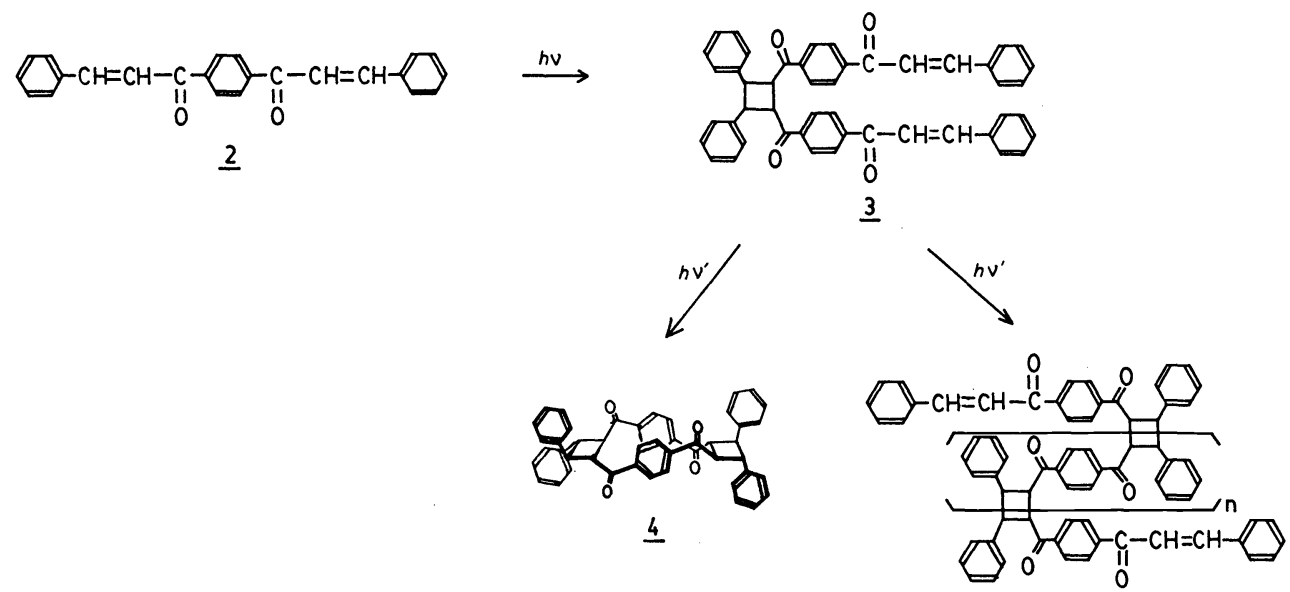

Oligomers 
topochemically, isolated in a preparative scale and identified. For example, on the photoirradiation of crystal 2 at room temperature gives $>90 \%$ of $\underline{4}$ (isolated in 58 \& yield after reprecipitation) and a small amount of oligomers.

Recent studies of the photochemical behavior of unsymmetric diolefin crystals have revealed several more deviations from the topochemical principle concerned with distance and the parallelism of double bonds described in following section.

\section{TOPOCHEMICAL BEHAVIOUR OF UNSYMMETRIC DIOLEFIN CRYSTALS}

Linear polymers derived from $\alpha$-type crystal of unsymmetric diolefins have a "hetero-adduct" or "homo-adduct" structure, as will be shown below.

One of the famous examples of hetero-adduct photoproducts is optically active oligomeric substances, which have been prepared through crystallization of achiral unsymmetric diolefin into a chiral crystal, followed by the fourcenter type photopolymerization (ref. 10).

Recently we have prepared and identified a great numer of unsymmetrically substituted photoreactive diolefin crystals with a general formula represented as (I)-(V), where $R$ and $R^{\prime}$ in groups (I) and (II) represent either hydrogen, methyl, ethyl or phenyl. In group (III) and (IV), $X$ is hydrogen or cyano group, and $\mathrm{Y}$ represents either cyano, alkoxycarbonyl or benzoyl groups. $R$ in group ( $V)$, which is unsymmetric DSP, is either alkoxycarbonyl or chlorine.

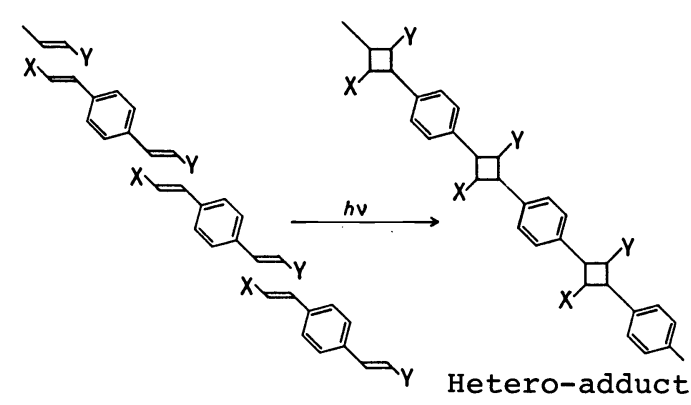

$\mathrm{ROOCCH}=\mathrm{CH}-\mathrm{CH}=\mathrm{CHCOOR}$<smiles>CCOC(=O)C=Cc1ccc(C=CCC(=O)c2ccccc2)cc1</smiles><smiles>[Y]C([X])=Cc1ccc(C=Cc2ccc[nH]2)cc1</smiles>

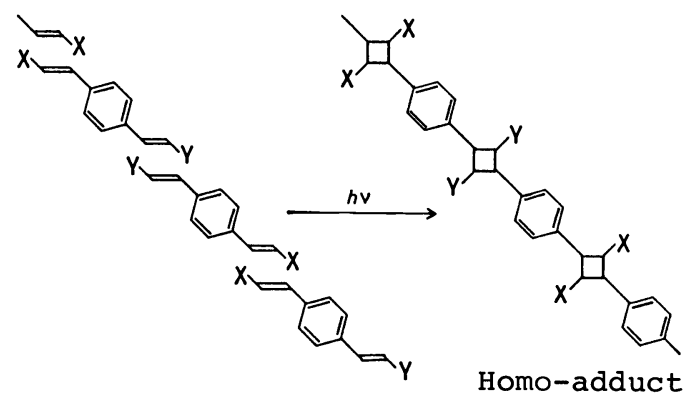<smiles>[X]C([Y])=Cc1ccc(C=Cc2ncc[nH]2)cc1</smiles>

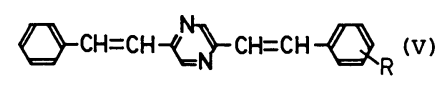

These crystals prepared so far show a prominent photoreactivity with a few exceptions. Several crystals are extremely highly photoreactive and, on photoirradiation, are converted into crystalline or amorphous high polymers with 1,3-trans cyclobutane ring in the main chain, or into photostable dimers.

For example, methyl 4-[2-(2-pyrazyl)ethenyl]cinnamate (IV, $\mathrm{X}$ is hydrogen and $Y$ is methoxycarbonyl) crystal gives a linear high polymer crystal ( $n$ inh 8.2 $\mathrm{dL} / \mathrm{g}, 0.3 \mathrm{~g} / \mathrm{dL}$ in 2 -hexafloropropanol at $30^{\circ} \mathrm{C}$ ) on photoirradiation by a 100 W high-pressure mercury lamp for $5 \mathrm{~h}$, or ever under sunlight for a day. Unsymmetric DSP crystal ( $V, R$ is methoxycarbonyl) gives a crystalline linear high polymer ( $n$ inh $1.3 \mathrm{dL} / \mathrm{g}, 0.30 \mathrm{~g} / \mathrm{dL}$ in 2-hexafloropropanol at $30^{\circ} \mathrm{C}$ ) quantitatively on the photoirradiation. Even in the reactions which give amorphous products, the topochemical control is obvious because a single configuration of cyclobutane is formed exclusively in all the resulting products. 
a Examples of hetero-adduct photoproduct

photostable dimer have been prepared from respectively.
A linear high polymer and a $\alpha$ and $\beta$-type packing crystals,

From spectroscopic and crystallographic analysis, it is concluded that on irradiation, the crystal of methyl 4-[2-(4-pyridyl)ethenyl]cinnamate ( $\underline{5})$, (III, 4-pyridyl, $\mathrm{X}$ is hydrogen and $\mathrm{Y}$ is methoxycabonyl) gives an amorphous heteroadduct polymer 6 in which a cyclobutane ring has the 1,3-trans configuration. The polymer is soluble in $\mathrm{N}, \mathrm{N}$-dimethylformamide, dimethylsulfoxide and strong acids such as concentrated hydrochloric acid ( $\mathrm{n}$ inh $0.47 \mathrm{dL} / \mathrm{g}, 0.30 \mathrm{~g} / \mathrm{dL}$ in $\mathrm{m}$-cresol at $30^{\circ} \mathrm{C}$ ), indicating the electrolyte character of the polymer.

The crystal structure of 5 viewed along the a axis is shown in Fig. 2 (ref. $14(\mathrm{~b}))$.

N- $-\mathrm{CH}=\mathrm{CH}-2-\mathrm{CH}=\mathrm{CHCOOMe}$

$\underline{5}$

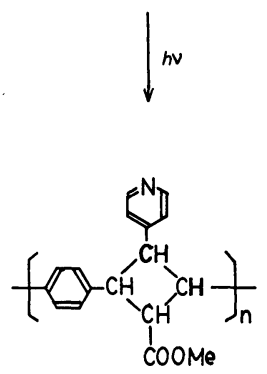

$\underline{6}$

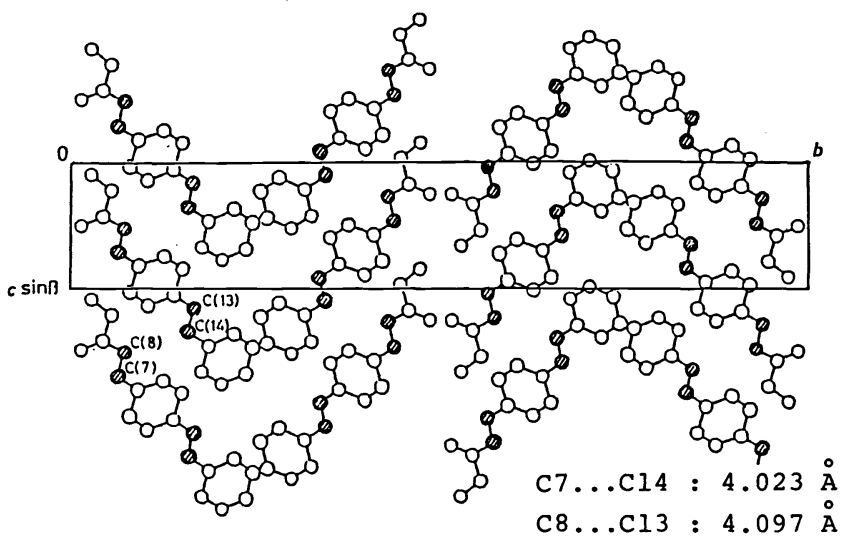

Fig. 2. Crystal structure of 5 viewed along a-axis. The atoms comprising the ethylenic groups are hatched for clarity.

The intermolecular reactive double bonds, separated by 4.023 (9) $\AA$ for $C(7) \cdots C(14)$ and $4.097(1) \AA$ for $C(8) \cdots C(13)$, are nonparallel, while these distances are within those of normal reactive bonds. The I-dimer-type racemic structure of 6 coincides with the structure of the topochemical photoproduct derived from $\overline{5}$, as the molecules are related to centrosymmetry and as the molecular columns are aligned in alternating directions in the crystal.

The crystal of methyl $\alpha$-cyano-4-[2-(2-pyridyl)ethenyl]cinnamate (7) (III, 2pyridyl, $X$ is cyano and $Y$ is methoxycarbonyl) is highly reactive, but, on photoirradiation, gives a photostable amorphous dimer (8) quantitatively.

As is illustrated in Fig. 3 (ref. $14(\mathrm{c})$ ), in the crystal 7 the reactive double bonds, separated by 3.886 (7) $\AA$ for $C(7) \cdots C\left(15^{\prime}\right)$ and 3.807 (7) $\AA$ for $c(8) \cdots c\left(16^{\prime}\right)$, are non-parallel, while these distances are within those of normal reactive bonds. Other distances of intermolecular olefin bonds are $4.926(7) \AA$ for $C(7) \cdots C\left(15^{\prime \prime}\right)$ and $4.719(6) \AA$ for $C(7) \cdots C(8 ")$.

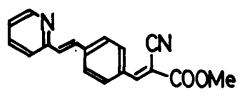

I<smiles>[CH]1CCC1</smiles>

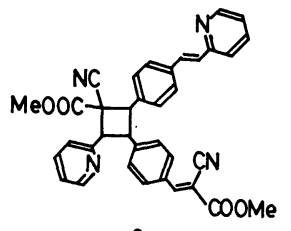

$\underline{8}$
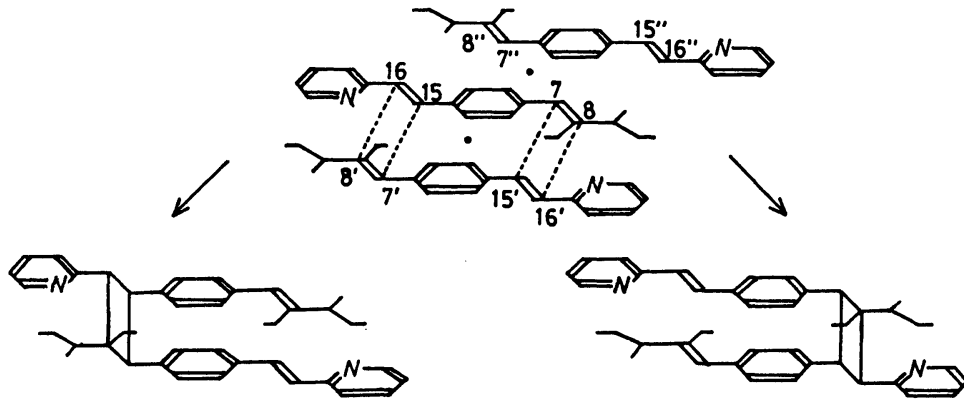

$\begin{array}{lll}C 8 \ldots C 16^{\prime}: & 3.807 & (7) \AA \\ C 7 \ldots C 15^{\prime}: & 3.886 & (7) \AA\end{array}$

Fig. 3. Schematic illustration of the formation of racemic dimer $\underline{8}$ from $\underline{7}$ 
As residual pair of double bonds will be rearranged far beyond the photoreactive distance in the dimer crystal, highly photostable behavior of the dimer 8 is reasonable. As the center of symmetry exists between two monomer molecules arranged in $\beta$-form, and as two pairs of double bonds in the monomer should be equivalent in reactivity, either enanthiomers are formed with the same probability.

\section{b Examples of homo-adduct photoproduct}

The crystals which afford the homo-adduct products, have generally two pairs of double bonds with different topochemical reactivity. Therefore, these crystals generally afford a single type of the dimers. The resultant dimers are photoreactive to give the high polymer on further photoirradiation, or are photostable.

Ethyl methyl 1,4-phenylenediacrylate (9), ( $I, R$ and $R^{\prime}$ are ethyl and methyl, respectively) photopolymerizes into a linear polymer (11) in the crystalline state. However, a large amount of dimer (10) is formed exclusively and accumulated at the intermediate stage of polymerization. On the other hand, no other types of the dimer are detected during the whole reaction process.

By MS and NMR spectroscopy 10 was confirmed to have the cyclobutane structure in which two ethyl cinnamate and two methoxycarbonyl groups are alternatively substituted. Exclusive photocyclodimerization between two olefins of methyl cinnamate groups in 9 is reasonably explained only by different topochemical circumstances between ethyl and methyl cinnamate groups. Both 10 crystals, as-prepared and recrystallized from methanol, are photoreactive. In the 9 crystal the molecules are arranged in parallel and related to the center of symmetry. The distances of intermolecular double bonds are 3.891 (6) $\AA$ between two methyl cinnamate groups (A in Fig. 4) and 4.917 (6) $\AA$ between two ethyl cinnamate groups ( $\mathrm{B}$ in Fig. 4), respectively. The distance between double bonds of ethyl and methyl cinnamate groups is approximately $6 \AA$ (C in Fig. 4) (ref. 26).

From the crystal structure of 9 exclusive formation of 10 from $^{-9}$ in the crystal is reasonably understood, although the distance between reactive double bonds of ethyl cinnamates in 10 has not yet been established.

Alkyl a-cyano-4-[2-(4pyridyl)ethenyl] ciannamate (III, X is cyano and $Y$ is alkoxycarbonyl, where alkyl is one of methyl, ethyl or $n$-propyl) shows a great variety of photochemical behavior in the crystalline state, depending on its alkyl species or recrystallizing solvents. Topochemical behavior of the ethyl ester (12) is similar to that of 9 : only one type of photoreactive dimer (13) is
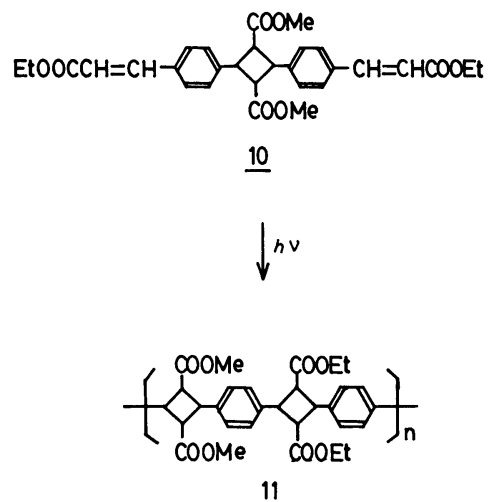

$\underline{10}$

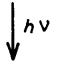

11 accumulated at the intermediate and, on further photoirradiation, is converted into a linear homo adduct polymer (14). In the crystal of 12 , every molecule is related to its neighboring molecules by two different centers of inversion along the stack direction. Two pairs of double bonds are separated by a distance of 3.758 (8) $\AA$ and 4.878 (1) $\AA$, respectively (ref. $14(\mathrm{~b})$ ), as shown in the scheme over leaf. 
As the former distance is within the conventional reactive one, while the latter is longer than the reactive one, the dimerization occurs exclusively between the former double bonds.

In consequence, 12 gives a linear homo adduct polymer 14 via the accumulation of the photoreactive dimer 13 .

No growing species having odd-numbered degree of polymerization, such as trimer or pentamer, have been detected at all in GPC curves at intermediate stages of reaction, as is illustrated in Fig. 6 .

The GPC study indicates that the monomer 12 reacts only with other monomer and then all the reacting species have evennumbered degree of polymerization during the stepwise topochemical process.

"Even-numbered Polymerization Mechanism" $\mathrm{M}+\mathrm{M} \stackrel{h \nu}{\longrightarrow}$ Dimer $(\mathrm{M}-\mathrm{M}) \stackrel{h \nu}{\longrightarrow}$ Polymer $(\mathrm{M}-\mathrm{M}) \mathrm{n}$

In addition, in the even-numbered polymerization mechanism, all the reactive species in crystal 12 should have pyridyl ethenyl groups at growing terminal.

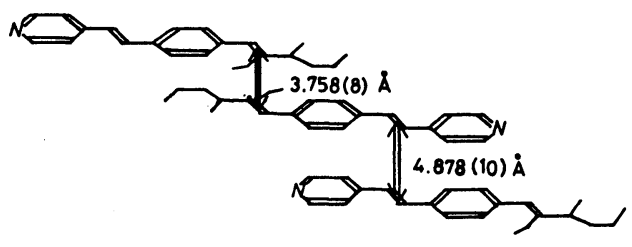

Fig. 5. Schematic illustration of the exclusive formation of the dimer 13 from 12

In contrast to the reaction of 12 , the GPC curve in the topochemical reaction of $\underline{9}$ shows a small but definite peak of trimer, indicating the reaction between 9 and 10 in the crystal. 13 Recrystallized from ethanol, exhibits essentialily the same crystal diffraction pattern with the crystal 13 asprepared, but photoirradiation, gives a higher-molecular-weight polymer, 14 $\left(n i n h=0.36 \mathrm{dL} / \mathrm{g} \rightarrow \eta \mathrm{inh}=0.64 \mathrm{dL} / \mathrm{g} \mathrm{m-cresol,} 30^{\circ} \mathrm{C}\right)$.

From the same methyl ester monomer, the same type of homo adduct polymer as 14 is prepared, while the accumulated dimer has quite the reverse structure of cyclobutane in which pyridyl side olefins first dimerized.

The same n-propyl ester is as highly as photoreactive with 12, however, gives photostable dimer quantitatively. The dimer, recrystallized from ethyl acetate, shows essentially the same X-ray diffraction pattern with asprepared. On the other hand,' recrystallized from n-propanol, shows different pattern and polymerizes on photoirradiation, into the homo adduct polymer ( $n$ inh $\left.0.87 \mathrm{dL} / \mathrm{g}, \mathrm{CHCl}_{3} 30^{\circ} \mathrm{C}\right)$.

The overall reaction behavior of alkyl $\alpha$-cyano-4-[2-(4-pyridyl)ethenyl]cinnamates is summarized in the scheme below.

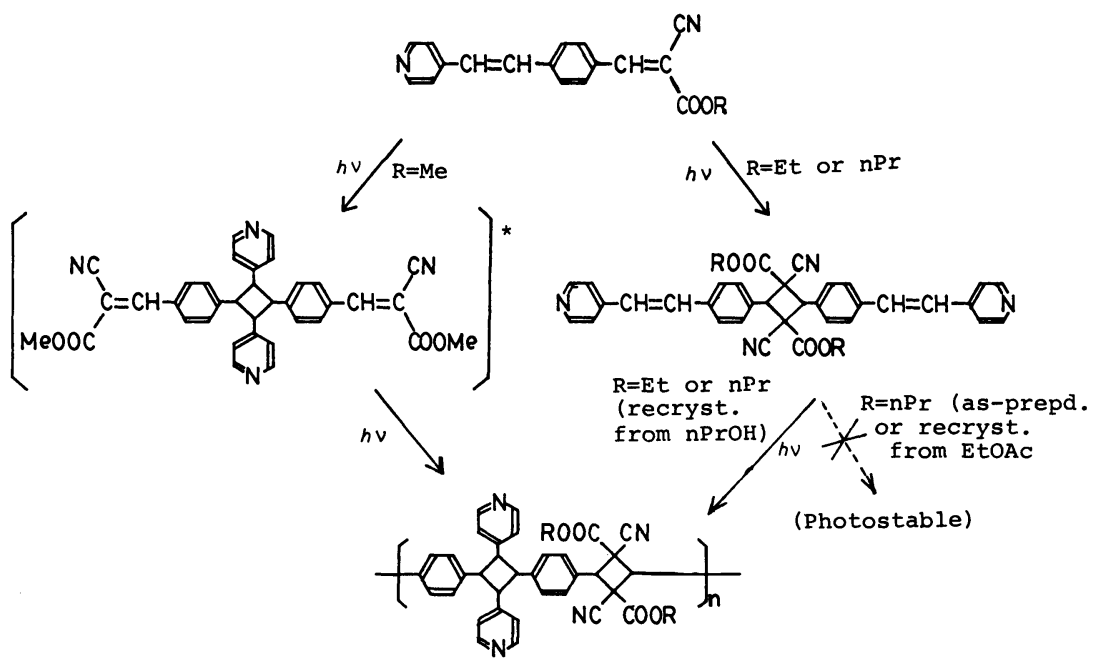




\section{CONCLUSIONS}

Topochemical [2+2] photocyclodimerizations are unique in that the crystal symmetry is retained throughout all the reaction processes. Linear high polymers, dervied from the four-center type photopolymerization of conjugated diolefin crystals, have a structure of a rigid rod-like extended chain. These polymers are extremely highly crystalline, while being aggregates of tiny single crystallites. Another example of topochemical polymerizations is the polymerization of conjugated diacetylene crystals in which a giant polymer single crystal is produced via a crystal lattice controlled chain mechanism (ref. 27).

Several [2+2] photocyclodimerizations of the crystals deviate from the topochemical principle. However, in almost all of these examples the topochemical concept established by schmidt is preserved because these products are formed with a minimum of atomic and molecular motion, causing these configurations to coincide with those predicted from the crystal structures of starting compounds.

From unsymmetric

diolefin crystals,

optically active

polymers or the

polymers with

hydrophobic and

hydrophilic units in

alternative order in

polymer chain (Fig. 7)

can be prepared.

The polymerization of unsymmetric diolefin crystals sometimes proceeds under the "even-numbered polymerization mechanism". Most of the polymers prepared by four-center type photopolymerization, are easily depolymerized photochemically and thermally in solution to the corresponding monomers, as is to be expected from the ring-cleavage reaction of a number of cyclobutane derivatives yielding two olefins (ref. 28, 29). One of the exceptional, anomalous examples is the degradation of poly-DSP. Anomalous poly-DSP is rapidly photooxidized in the air upon exposure to sunlight and produces nitrile derivatives, together with products containing carbonyl and hydroxyl groups, suggesting the degradation of the pyrazine ring (ref. 30). The structural trigger of the photooxidation has not yet been characterized, although a great effect of acidic media on the nitrile formation has been recognized. Several recent articles deal with the application of these unsymmetric crystals to LB-film techniques (ref. 3l) and to the non-linear optics (ref. 32).

The correlation of the crystal structure of starting compounds with their chemical structures is very subtle; in addition, these crystal structures, which in turn influence topochemical reactivity, are, in some cases, variable with the crystallization process and with the recrystallizing solvents used. Further studies of the relation between chemical structure and topochemical reactivity, are required to reveal the whole shape of the crystal engineering concept.

\footnotetext{
Acknowledgements - The author is grateful to his colleagues Associate Professor Dr. Kazuhiko Saigo, Dr. Nobuyuki Yonezawa, Mr. Masao Nohara, Mr. Tohru Mori, Mr. Hirobumi Uno, Mr. Hatsuhiko Harashina and Mr. Satoshi Kato (The University of Tokyo), for their valuable contributions to this work. He is also pleased to acknowledge the help of Dr. Hachiro Nakanishi, Dr. Sadao Sato and Dr. Kazuhide Kamiya in the crystallographic analysis.
}

\section{REFERENCES}

1. Liebermann, C. Chem. Ber. 22, 124, 782 (1889).

2. Bernstein, H. I.; Quimby, W. C. J. Am. 드르. 므으. $6 \underline{5}, 1845$ (1943). 
3. Cohen, M. D.; Schmidt, G. M. J. J. Chem. Soc. 1969 (1964); for a review of "Topochemical [2+2] photocyclodimerization", see Schmidt, G. M. J. Pure Appl. Chem. 27, 647 (1971).

4. Hasegawa, M.; Suzuki,Y. J. Polym. Sci. B 5 , 813 (1967).

5. Nakanishi, H.; Hasegawa, M.; Sasada, Y. J. Polym. Sci. Polym. Chem. Ed. 10,1537 (1972).

6. Nakanishi, H.; Ueno, K.; Sasada, Y. Acta Cryst. B32, 3352 (1986).

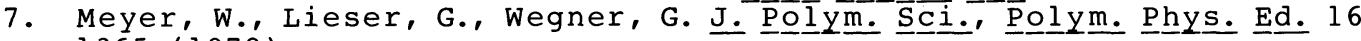
$1365(1978)$.

8. Nakanishi, H., Sasada, Y., Hasegawa, M. Polyym. Lettt. 17, 459 (1979).

9. Nakanishi, F.; Hasegawa, M. J. Polym. Sci., Polym Chem. Ed. 8, 2151 (1970); Hasegawa, M. et a l.; Progr. Polym. Sci.' Jpn. $5,143(1973)$.

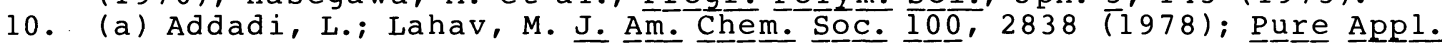
Chem. 51, 1269 (1979).

(b) Ädadi, L.; Van Mil, J.; Lahav, M. A. J. Am. Chem. Soc. 104, 3422 (1982).

11. Nakanishi, H.; Hasegawa, M.; Sasada, Y. J. Pollym. Sci., Pollym. Phys. Ed. $15,173(1977)$.

12. The review articles of the four-center type photopolymerization

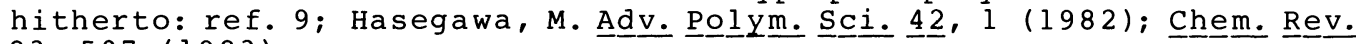
$83,507(1983)$.

13. For a review of the results: Hasagawa, M.; Saigo, K.; Mori, T. ACS Symp. ser. $266,255(1984)$.

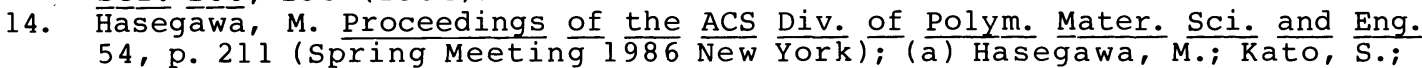
Yonezawa, N.; Saigo, K. J. Pollym. Sci., Pollym. Lett. Ed. in press; (b) Hasegawa, M.; Harashina, H.; Kato, S.; Saigo, K. Macromolecules in press;(c) Kato, S.; Nakatani, M.; Harashina, H.; Saigo, K.; Hasegawa, M.; Sato, S. Chem. Lett. submitted.

15. Ramasubbe, N.; Guru Row, T. N.; Venkatesan, K.; Ramamurthy, V.; Rao, C. N. R. J. Chem. Soc., Chem. Commun. 178 (1982).

16. Theocharis, C. R.; Jones, W.; Thomas, J. M.; Motevalli, M.; Harsthouse, M. B. J. Chem. Soc. Perkin Trans. II, 71 (1984).

17. Gnanaguru, K.; Ramasubbu, N.; Venkatesan, K.; Ramamurthy, V. J. Photochem. 27, 355 (1984); J. Org. Chem.50,2337 (1985).

18. Pfoentner, K. H.; Englart, G.; Schoenholger, P. XIIth International Conf. on Photochem. Preprint, p.487 (Tokyo, Jpn. Aug. 1985).

19. Desiraju, G. R.; Sarma, J. A. R. P. J. Chhem sooc., Chee․ Coommun. 45

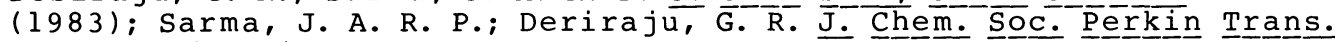
II, 1905 (1985).

20. Nakanishi, H.; Hasegawa, M.; Mori, T. Acta Cryst. C441, 70 (1985).

21. Hasegawa, M.; Arioka, H.; Harashina, H.; Nohara, M.; Kubo, M.; Nishikubo, T. Isr. J. Chem. 25, 302 (1985).

22. Hasegawa, M.; Saigo, K.; Mori, T.; Uno, H.; Nohara, M.; Nakanishi, H.; J. Am. Chem. Soc. 107, 2788 (1985).

23. Nakanishi, F.; Nakanishi, H.; Tsuchiya, M.; Hasegawa, M. Bull. Chem. Soc. Jpn. 49, 3096 (1976).

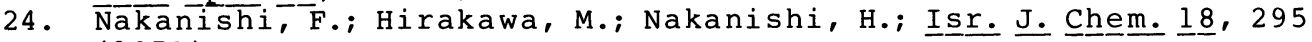
(1979).

25. (a) Leonard, J. K.; McCredie, R.S.; Longue, M. W.; Cundall, R. C. J. Am. Chem. Sooc. 95, 2320 (1973). (b) Frank, J. K.; Paul, I. C. J. Am. Cheme 드으. 9 드.

26. Crystal data of 5: PI, $a=12.460(1) \AA, b=9.713(1) \AA, c=5.958(1) \AA$, $\alpha=77.43(1)^{\circ}, \beta=87.09(1)^{\circ}, \gamma=80.75(1)^{\circ}, Z=2, V=694.5 \mathrm{~A}, \mathrm{R}=$ 0.069 .

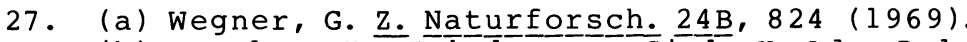

(b) Bassler, H. ; Enkelmann,i Sixl, H. Adv. Polym. Sci. 63 (1984).

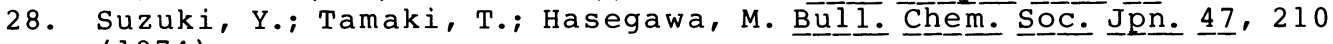
(1974).

29. Hasegawa, M.; Nakanishi, H.; Yurugi, T. Polym. Lett. 14, 47 (1976).

30. Sakuragi, M.; Hasegawa, M.; Nishigaki, M. J. Pollym. Sci., Polym. Che Ed. $14,521(1976)$.

31. Nakanishi, F.; Okada, S.; Ichimura, K.; Suda, M. Annual Symposium Preprint of Res. Inst. for Polym. and Text. p. 159 (Nov. 1985).

32. Miyata, S. et. al, private communication, to be published. 